Fetal Diagnosis and Therapy
Fetal Diagn Ther 2017;42:144-149

DOI: $10.1159 / 000456615$
Received: September 16, 2016

Accepted after revision: January 5, 2017

Published online: March 4, 2017

\title{
Congenital Cytomegalovirus Infection in the Absence of Maternal Cytomegalovirus-IgM Antibodies
}

\author{
Julia Gunkel ${ }^{a}$ Bloeme J. van der Knoop ${ }^{a, f}$ Joppe Nijman ${ }^{a}$ Linda S. de Vries ${ }^{a}$ \\ Gwendolyn T.R. Manten $^{b}$ Peter G.J. Nikkels ${ }^{c}$ Jean-Luc Murk ${ }^{d}$ \\ Johanna I.P. de Vries ${ }^{a, f}$ Tom F.W. Wolfs ${ }^{e}$ \\ Departments of a Neonatology, ${ }^{\mathrm{b}}$ Perinatology, ${ }^{\mathrm{c} P a t h o l o g y,}{ }^{\mathrm{d}}$ Medical Microbiology, and ${ }^{\mathrm{e}}$ Pediatric Infectious \\ Diseases, University Medical Center Utrecht, Utrecht, and f Department of Obstetrics and Gynecology, VU University \\ Medical Center, Amsterdam, The Netherlands
}

\section{Keywords}

Cytomegalovirus · Congenital infection · Fetal ultrasound abnormalities $\cdot$ Maternal primary infection $\cdot$ Maternal non-primary infection $\cdot$ Outcome

\begin{abstract}
Background: Congenital cytomegalovirus (cCMV) infections are the most prevalent intrauterine infections worldwide and are the result of maternal primary or non-primary infections. Early maternal primary infections are thought to carry the highest risk of fetal developmental abnormalities as seen by ultrasound; however, non-primary infections may prove equally detrimental. Methods/Results: This case series presents 5 cases with fetal abnormalities detected in the second and third trimester, in which CCMV infection was ruled out due to negative maternal CMV-IgM. Discussion: This series highlights the possible pitfalls in serology interpretation and fetal diagnosis necessary for appropriate parental counseling. Once fetal abnormalities have been confirmed and CCMV is suspected, maternal CMV serostatus and fetal infection should be determined. Maternal CMV serology may be ambiguous; therefore, caution should be exercised when interpreting the results.

(c) 2017 The Author(s)

Published by S. Karger AG, Basel
\end{abstract}

\section{KARGER}

E-Mail karger@karger.com www.karger.com/fdt
This article is licensed under the Creative Commons AttributionNonCommercial-NoDerivatives 4.0 International License (CC BYNC-ND) (http://www.karger.com/Services/OpenAccessLicense). Usage and distribution for commercial purposes as well as any distribution of modified material requires written permission.

\section{Introduction}

Congenital cytomegalovirus (cCMV) infections occur frequently with a birth prevalence of around $1 \%$, of which $11 \%$ of the infants will be symptomatic at birth [1]. Transmission of CMV can occur due to a maternal primary infection in previously seronegative women or after a non-primary infection (reactivation of an endogenous strain or reinfection with a new CMV strain) in women with preconceptional immunity [2]. Vertical transmission to the fetus during a maternal primary infection occurs in about $30 \%[3,4]$ and during non-primary infections in about $1-2 \%$ of pregnancies [1], although this rate may be higher [2]. Maternal primary infections early in pregnancy occur less frequently but are thought to carry the highest risk of fetal abnormalities as seen by ultrasound (US) and/or symptomatic disease at birth [3, 4]. This has not been established for non-primary infections. CMV-associated fetal US abnormalities are broadly defined as cerebral or extracerebral and can be transient, non-specific, and may occur in any trimester. Extracere-

\section{J. Gunkel and B.J. van der Knoop share first authorship.}

Tom F.W. Wolfs, MD, PhD

Department of Pediatric Infectious Diseases

Wilhelmina Children's Hospital, University Medical Center Utrecht

PO Box 85090, NL-3508 AB Utrecht (The Netherlands)

E-Mail T.Wolfs@umcutrecht.nl 
bral US abnormalities include intrauterine growth restriction, hydrops, hepatomegaly, and echogenic bowel [5]. Cerebral US anomalies can be mild (lenticulostriate vasculopathy [LSV], germinolytic cysts, mild ventricular dilatation, periventricular echogenicity) or more severe (cystic lesions in the white matter, moderate to severe ventricular dilatation, cerebellar hypoplasia, polymicrogyria and lissencephaly, microcephaly) [6-8]. In the presence of cerebral abnormalities, there is a high risk of adverse neurodevelopmental outcomes [7, 9]. Primary infections are recognized by $\mathrm{CMV}$-immunoglobulin $\mathrm{G}$ (IgG) seroconversion or positive CMV-immunoglobulin M (IgM) with a low IgG avidity index (AI) [1]. When looking at CMV-IgM kinetics following primary infection, peak levels are seen in the first 1-3 months, after which the titers begin to decrease [10]. Occasionally, persistent (low) levels of CMV-IgM can be detected $>3$ months or up to a year [10]. Non-primary infections are difficult to diagnose but may be recognized by positive CMV-IgG prior to conception/early gestation in combination with a positive CMV-IgM and CMV-IgG with a high IgG AI and/or a significant increase in CMV-IgG titer during gestation $[1,10,11]$. When the type of maternal infection cannot be classified on the basis of CMVIgM, CMV-IgG AI may aid in distinguishing between primary and non-primary infection $[12,13]$. A high IgG $\mathrm{AI}$ is usually seen around 5-6 months following primary infection. Dating the timing of the infection through maternal serology is difficult, and interpretation of the results is not always straightforward, especially because samples are often collected long after the maternal CMV infection has occurred. Here we report 5 cases with fetal anomalies in the second and third trimester, whereby cCMV infection was initially considered unlikely because maternal CMV-IgM was negative at the time of presentation.

\section{Case Presentation and Results}

\section{Case 1}

Case 1 was referred at 20 weeks' gestation (WG) because of fetal echogenic bowels. Amniocentesis and maternal serology testing for CMV and toxoplasmosis were offered but declined. Genetic carrier testing of both parents revealed no mutation on the CFTR gene, and therefore cystic fibrosis was considered unlikely. The US at 32 WG to monitor bowel development showed reduced echogenicity but now revealed extensive bilateral LSV (Fig. 1a). Maternal CMV serology was tested at this time

Antenatal Maternal Serology in

Congenital CMV Infection

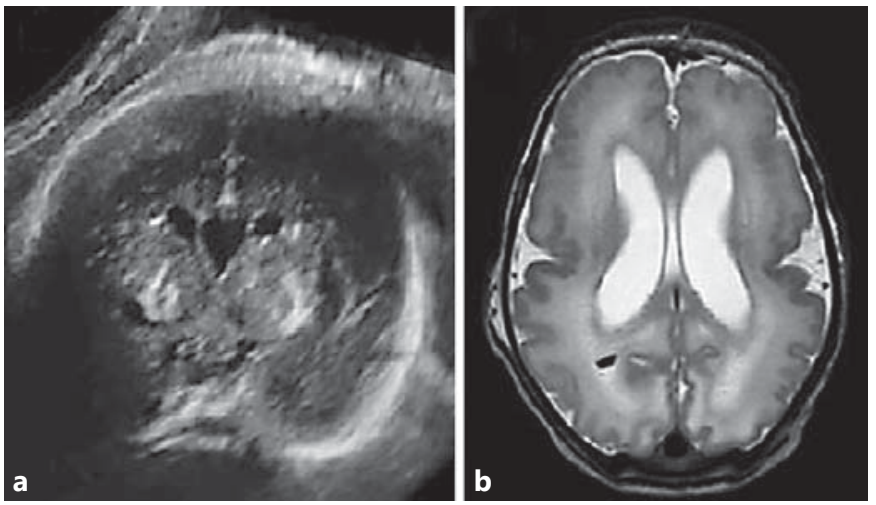

Fig. 1. a Case 1: fetal US at 32 weeks' gestation, coronal plane, showing bilateral LSV. b Case 5: MRI, axial T2-weighted sequence, showing ventriculomegaly, increased signal intensity throughout the white matter, a small hemorrhage in the white matter (right occipital), and bilateral extensive polymicrogyria.

and was indicative of CMV infection in the past (Table 1). At $40+1 \mathrm{WG}$ a female infant was born with a birth weight of $3,460 \mathrm{~g}$ (percentile [p] 45), a length of $52 \mathrm{~cm}$ (p50), a head circumference (HC) of $36 \mathrm{~cm}$ (p50), and Apgar scores of $8 / 10 / 10$. The infant had widespread petechiae, hepatosplenomegaly, and thrombocytopenia (44 $\times$ $10^{9} / \mathrm{L}$ ). Cranial US on day of life (DOL) 1 indicated extensive bilateral LSV, white matter calcifications, and bilateral germinolytic cysts. Urine CMV polymerase chain reaction (PCR) tested positive, confirming cCMV infection. Hearing and ophthalmological tests were normal. The infant was treated with valganciclovir for 6 weeks. MRI performed at 5 months of age showed white matter signal intensity changes and resolution of the germinolytic cysts. First trimester maternal serum was tested in retrospect and was indicative of a primary CMV infection (Table 1). The Alberta Infant Motor Scale and Bayley Scales of Infant and Toddler Development (BSITD-III) at 12 months of age showed mild neurodevelopmental impairment.

\section{Case 2}

Case 2 was referred at $30+2$ WG due to mild bilateral ventriculomegaly. This was a repeat US after a routine US at $20 \mathrm{WG}$ showed mild hydronephrosis, which had now normalized. Maternal serology was negative for toxoplasmosis and indicated CMV infection in the past (Table 1). The dilatation was initially progressive and eventually stabilized at $35 \mathrm{WG}$. At $38 \mathrm{WG}$ a female infant was born with a birth weight of $2,750 \mathrm{~g}(\mathrm{p} 20)$, a length of $54 \mathrm{~cm}$ (>p97), a HC of $33 \mathrm{~cm}$ (p20), Apgar scores of 8/9/10, and

Fetal Diagn Ther 2017;42:144-149 145 
Table 1. Characteristics of 5 fetuses with US findings suggestive of CMV infection and maternal serology

\begin{tabular}{|c|c|c|c|c|c|c|c|c|c|c|c|c|}
\hline \multirow[t]{2}{*}{ Case } & \multirow[t]{2}{*}{ Maternal } & \multirow[t]{2}{*}{$\begin{array}{l}\text { GA at first } \\
\text { presentation }\end{array}$} & \multirow[t]{2}{*}{ Fetal US anomalies } & \multirow[t]{2}{*}{$\begin{array}{l}\text { GA at } \\
\text { testing }\end{array}$} & \multicolumn{3}{|c|}{$\begin{array}{l}\text { CMV serology at time of } \\
\text { US }(2 \mathrm{~T} / 3 \mathrm{~T})\end{array}$} & \multirow[t]{2}{*}{$\begin{array}{l}\mathrm{GA} \text { at } \\
\text { testing }\end{array}$} & \multicolumn{3}{|c|}{ CMV serology at $1 \mathrm{~T}$} & \multirow[t]{2}{*}{$\begin{array}{l}\text { Interpre- } \\
\text { tation }\end{array}$} \\
\hline & & & & & $\operatorname{IgM}$ & $\operatorname{IgG}$ & avidity & & $\operatorname{Ig} M$ & IgG & avidity & \\
\hline 1 & G4P2 & 20 & $\begin{array}{l}\text { Echogenic bowels, LSV } \\
\text { (at GA 32) }\end{array}$ & 32 & - & + & $0.88^{\mathrm{a}}$ & 12 & + & + & $0.27^{\mathrm{a}}$ & $\begin{array}{l}\text { Primary } \\
\text { infection }\end{array}$ \\
\hline 2 & G2P1 & $30+2$ & $\mathrm{VM}$ & $32+4$ & - & + & $0.95^{\mathrm{a}}$ & n.a. & n.a. & n.a. & n.a. & Unknown \\
\hline 3 & G2P1 & $21+4$ & $\begin{array}{l}\mathrm{HC}<\mathrm{p} 3, \mathrm{FL}<\mathrm{p} 3 \text {, enlarged } \\
\text { heart, oligohydramnios, } \\
\text { echogenic bowels, FH, } \\
\text { thickened nuchal fold }\end{array}$ & $21+4$ & - & + & $0.74^{\mathrm{a}}$ & $8+3$ & - & + & n.a. & $\begin{array}{l}\text { Probable } \\
\text { primary } \\
\text { infection }\end{array}$ \\
\hline 4 & G2P1 & $21+6$ & HC $\mathrm{p} 3$, cerebellum $<\mathrm{p} 3$ & $21+6$ & - & + & $0.68^{\mathrm{a}}$ & 13 & + & + & $0.62^{\mathrm{b}}$ & $\begin{array}{l}\text { Non-primary } \\
\text { infection }\end{array}$ \\
\hline 5 & G1P0 & $22+2$ & $\mathrm{HC}<\mathrm{p} 3$, echogenic bowel & $22+2$ & - & + & $0.96^{\mathrm{a}}$ & 12 & - & + & $0.91^{\mathrm{a}}$ & $\begin{array}{l}\text { Non-primary } \\
\text { infection }\end{array}$ \\
\hline
\end{tabular}

CMV, cytomegalovirus; GA, gestational age in weeks + days; 1T, first trimester; 2T, second trimester; 3T, third trimester; IgM, immunoglobulin M; IgG, immunoglobulin G; HC, head circumference; FL, femur length; LSV, lenticulostriate vasculopathy; US, ultrasound; VM, ventriculomegaly; FH, fetal hydrops; n.a., not available.

a VIDAS, bioMérieux CMV IgG avidity index (AI) (high AI: >0.65, intermediate AI: 0.65-0.40, low AI: $<0.40$ ). ${ }^{\mathrm{b}}$ LIAISON XL, DiaSorin CMV IgG AI (high AI: $>0.25$, intermediate AI: $0.25-0.15$, low AI: $<0.15$ ).

no physical symptoms. Laboratory analysis revealed thrombocytopenia $\left(114 \times 10^{9} / \mathrm{L}\right)$, resolving spontaneously by DOL 8 . Cranial US performed on DOL 1 showed bilateral ventriculomegaly, bilateral LSV, germinolytic cysts, and a cyst in the right temporal lobe. MRI was carried out on DOL 2 and showed high signal intensity in the white matter, bilateral: germinolytic cysts, subependymal cysts, large temporal cysts, and small occipital cysts. Urine CMV PCR tested positive on DOL 2, confirming CCMV infection. Hearing and ophthalmological tests were normal. The infant was treated with valganciclovir for 6 weeks. First-trimester maternal serum could not be tested retrospectively as it was discarded. The Griffiths Mental Developmental Scales (GMDS) assessment at 36 months was within the normal range (developmental quotient 100 ), and her hearing was normal.

\section{Case 3}

Case 3 was referred at $21+4 \mathrm{WG}$ due to a $\mathrm{HC}<\mathrm{p} 3$, a femur length $<\mathrm{p} 3$, an enlarged heart, oligohydramnios, and echogenic bowels. Advanced US confirmed these findings and additionally revealed fetal hydrops and a thickened nuchal fold. Maternal serology was tested and was negative for toxoplasmosis, enteroviruses, parvovirus $\mathrm{B} 19$, and varicella zoster virus and showed CMV infection in the past (Table 1). Amniocentesis was performed at $22+4 \mathrm{WG}$ revealing a genetic duplication and deletion, which was also present in the mother and therefore not considered causative. CMV PCR was not carried out at this point. Fetal growth stagnated at $23+3 \mathrm{WG}$ with $\mathrm{HC}$ $<\mathrm{p} 3$ and an increase in fetal hydrops. Parents were counseled about the risk of a poor outcome and chose to terminate the pregnancy. At $23+6 \mathrm{WG}$ a male infant was born weighing $573 \mathrm{~g}$ (p10-20). Autopsy confirmed the pericardial effusion and ascites but not intrauterine growth restriction (HC p20-50, femur length p20-50). The heart had a dilated left ventricle and a small atriumseptum defect. Immunohistochemical staining was positive for CMV in the pancreas, spine, liver, lung, kidneys, and placenta, and severe cytomegalic inclusion bodies were found throughout the brain, confirming cCMV infection. Retrospective analysis after fetal autopsy of firsttrimester maternal serum and amniotic fluid revealed CMV infection in the past and a positive CMV PCR, respectively. Unfortunately, a CMV-IgG AI was not possible to determine as no serum was available for additional tests.

\section{Case 4}

Case 4 was referred at $21+6 \mathrm{WG}$ due to a $\mathrm{HC}<\mathrm{p} 5$. Advanced US revealed a HC at p3 and cerebellar hypoplasia (transcerebellar diameter $<\mathrm{p} 3$ ). Second-trimester maternal serology was negative for toxoplasmosis and indicated CMV infection in the past (Table 1). Amniocen-
146

Fetal Diagn Ther 2017;42:144-149 DOI: $10.1159 / 000456615$
Gunkel/van der Knoop/Nijman/de Vries/ Manten/Nikkels/Murk/de Vries/Wolfs 
tesis ruled out chromosomal abnormalities; CMV PCR was not performed. A repeat US at $22+5 \mathrm{WG}$ showed persistence of $\mathrm{HC}<\mathrm{p} 3$ and cerebellar hypoplasia $(<\mathrm{p} 3)$. Parents were counseled about the poor prognosis and decided to terminate the pregnancy. Autopsy revealed a female infant weighing $595 \mathrm{~g}$ (p20-50), with no exterior abnormalities. The small cerebellum was confirmed alongside a small brain (p5). Microscopic examination showed CMV inclusion bodies in the kidneys, pituitary gland, and throughout the cerebellum and cerebrum, confirming cCMV infection. Due to the discrepant results between second-trimester maternal serology and autopsy findings, first-trimester (in retrospect) and postpartum maternal serum were tested for CMV. First-trimester maternal serum revealed a possible non-primary infection with positive CMV-IgM and a high IgG AI (Table 1). Postpartum serology was the same as in the firsttrimester sample, however with an increase of the IgG AI.

\section{Case 5}

Case 5 was referred at $22+2 \mathrm{WG}$ due to a $\mathrm{HC}<\mathrm{p} 3$, echogenic bowels, and oligohydramnios. Advanced US confirmed these findings. Maternal serology was tested at $22+2$ WG revealing CMV infection in the past (Table 1) and no signs of toxoplasmosis, rubella virus, Treponema pallidum, or varicella zoster virus. Amniocentesis was declined, and the parents decided to continue the pregnancy. Throughout the pregnancy, HC and cerebellar growth remained below p3. At $37+4 \mathrm{WG}$ a female infant was born weighing 2,890 g (p20-50), with a length of $47 \mathrm{~cm}$ (p1020), a HC of $32 \mathrm{~cm}$ (p3), and Apgar scores of 7/7/9. The infant had widespread petechiae, purpura, hepatosplenomegaly, thrombocytopenia $\left(30 \times 10^{9} / \mathrm{L}\right)$, conjugated hyperbilirubinemia (total $455 \mu \mathrm{mol} / \mathrm{L}$, direct $>170 \mu \mathrm{mol} / \mathrm{L}$ ), and prolonged partial thromboplastin $(25.0 \mathrm{~s})$ and activated thromboplastin time (51 s). Cranial US on DOL 1 showed mild bilateral ventriculomegaly, a smooth aspect of the cortex, and bilateral LSV. MRI was performed on DOL 3 showing extensive polymicrogyria, intraventricular hemorrhage, subdural hemorrhage, supra- and infratentorial hemorrhagic lesions in the white matter, and multiple punctate hemorrhages in the cerebellum (Fig. 1b). Urine CMV PCR on DOL 1 was positive, confirming cCMV infection. Ophthalmological examination revealed lesions suggestive of chorioretinitis in one eye. Hearing was not tested. Her clinical condition deteriorated on DOL 3. Due to the poor prognosis, intensive care was not intensified and the infant died. Postmortem examination was declined. First-trimester serum was retrospectively tested and indicated CMV infection in the past (Table 1).

Antenatal Maternal Serology in

Congenital CMV Infection

\section{Discussion}

This series describes fetal anomalies suggestive of CMV infection, whereby maternal serology at the time of anomaly detection was CMV-IgM negative. Vertical CMV transmission was therefore considered unlikely in all cases. In the Netherlands, pregnant women are not screened for CMV but are routinely tested in the first trimester for HIV, syphilis, and hepatitis B. At 20 WG a routine sonogram is offered. When abnormalities are found, women are referred for an advanced US and, when needed, further diagnostics such as maternal serology testing and/or amniocentesis are offered. A pre- and postnatal national guideline is available when $\mathrm{CMV}$ infection is suspected. In practice, however, when maternal serology is suggestive of a CMV infection in the past due to absence of CMV-IgM, the possibility of vertical transmission is often ruled out. This series highlights the inhomogeneous presentation of maternal CMV serology, stresses the need for cautious interpretation, and warrants multiple diagnostic steps [14]. In all cases, the diagnosis of cCMV infection was made postpartum due to infant symptomatology or fetal autopsy findings. A crucial albeit obvious learning point lays in the fact that in all cases maternal CMV serology was only tested once when anomalies were first detected. Intrauterine infections are an important differential group when fetal anomalies arise and are frequently tested by examining maternal serology for the TORCH complex (toxoplasma, other infectious pathogens such as rubella virus, cytomegalovirus, and herpes viruses). When considering CMV serology, however, factors such as gestational age at testing are essential to correct interpretation. Despite extensive literature on the topics of maternal CMV serology [for reviews, see 12,14$]$ and CMV-induced fetal anomalies $[4,5,12]$, in our experience the awareness of CMV as potential cause of fetal anomalies has not sufficiently penetrated clinical practice $[15,16]$.

For a correct interpretation of maternal CMV serology, it is ideal to know the CMV serostatus antecedent to pregnancy. Since this is often unknown, serum samples from standard screening (HIV, syphilis, and hepatitis B) in the first trimester should be saved to enable retrospective analysis of potential early primary infection. Frequently, serum samples are discarded immediately, making retrospective analysis not possible. Unfortunately, this was the case in case 2 , and therefore it was not possible to discern the type of maternal infection. The serology in case 1 exhibits the classic characteristics of a primary infection with low IgG AI and a positive CMV-IgM

Fetal Diagn Ther 2017;42:144-149 147 
in the first trimester. When symptoms are evident in the child after birth, we advise to immediately (no later than $\leq 3$ weeks postpartum) perform CMV PCR on the infants' urine to determine if vertical transmission has occurred. Case 3 may also exhibit a primary infection despite negative CMV-IgM in the first trimester. At $8+3 \mathrm{WG}, 2$ months have passed in which CMV-IgM could have already dropped below the detection limit [11]. Unfortunately, not enough serum was available for a first-trimester IgG AI test. However, an IgG AI of 0.74 (VIDAS, bioMérieux, high IgG AI >0.65) at $21+4$ WG could support an early primary infection. The IgG AI is low during the first 3-4 months after primary infection, followed by an intermediate IgG-AI for 1-2 months and subsequently full IgG avidity maturation, fitting this timeline [11]. In both cases, the decision to terminate the pregnancy may have not been altered; however, we believe that when clinicians choose to test for CMV that this should be done correctly to accurately counsel parents.

It was previously thought that non-primary infections have a low transmission rate due to preconceptional immunity. However, more recently, it has been noted that cCMV infections could occur more often in infants of preconceptionally seropositive women [for a review, see $2,17]$. Severe CMV-associated symptoms in the fetus/infant, as a result of non-primary maternal infection, have been reported [18] and are the probable cause in cases 4 and 5. Serological characteristics of non-primary infections remain elusive [19]; however, when fetal anomalies are present and maternal serology indicates non-primary infection, it is important to realize that cCMV infection may still have taken place. Regardless of the presence of CMV-IgM, a high IgG AI reflects immunological maturity and, encountered early on in gestation, pleads against first-trimester primary infection [14, 19]. Unfortunately, not enough serum was available for a retrospective CMVIgM analysis in case 4; however, the very high IgG AI at $11+5 \mathrm{WG}$ points to a non-primary infection regardless of CMV-IgM. When interpreting IgG AI amongst different laboratories, it is important to be aware of different AI cutoff values between assays (case 4) [13]. To ensure diagnostic accuracy, it is advisable to test all samples in one laboratory. IgG AI testing in case 4 was done in different laboratories due to referrals.

A useful gestational management scheme for CMV infection is proposed by Lazzarotto et al. [14], but since maternal CMV screening is not felt justified in most countries, a frequent diagnostic starting point are fetal anomalies seen on routine $20 \mathrm{WG}$ sonograms. Fetal infection can be diagnosed by CMV PCR of amniotic fluid and has been shown to have good sensitivity and a low risk of fetal loss $(<1 \%)$ when carried out after $20-21$ WG and $\geq 6-8$ weeks after the onset of maternal infection (if known) [4, $10,14]$. When amniocentesis is performed for other diagnostics (i.e., quantitative fluorescence PCR), we recommend to concurrently perform CMV PCR to investigate fetal CMV infection. This would have accelerated the diagnosis in cases 3 and 4 . Despite the fact that the clinical course may not have changed significantly, we want to stress the importance of CMV as a causative agent of fetal abnormalities and that when testing is done, this should be carried out at the correct time points and interpreted with sufficient expertise.

In conclusion, multiple diagnostic steps should be carried out to diagnose fetal cCMV infection. When fetal US anomalies are detected, referral for an advanced US should take place. Both maternal first- and second-/thirdtrimester serum should be tested for evidence of a primary infection. Storage of first-trimester serum should be obligatory to enable this. In the case of negative CMVIgM, a non-primary infection cannot always be excluded. If the mother is seronegative, cCMV infection can be excluded. To confirm fetal infection, amniocentesis can be offered. When cCMV infection is confirmed and the extent of cerebral and extracerebral abnormalities is determined, parents can be counseled accordingly.

\section{Acknowledgment}

Dr. J. Gunkel was financially supported by the Dutch Phelps Foundation (Grant number: 2012 - 020; http://www.phelps-stichting.nl).

\section{Statement of Ethics}

Consent was waived due to the use of anonymous data as part of standard care.

\section{Disclosure Statement}

The authors declare no conflict of interest.
148

Fetal Diagn Ther 2017;42:144-149 DOI: $10.1159 / 000456615$
Gunkel/van der Knoop/Nijman/de Vries/ Manten/Nikkels/Murk/de Vries/Wolfs 


\section{References}

1 Kenneson A, Cannon M: Review and metaanalysis of the epidemiology of congenital cytomegalovirus (CMV) infection. Rev Med Virol 2007; 17:253-276.

2 Britt W: Controversies in the natural history of congenital human cytomegalovirus infection: the paradox of infection and disease in offspring of women with immunity prior to pregnancy. Med Microbiol Immunol 2015; 204:263-271.

3 Enders G, Daiminger A, Bäder U, Exler S, Enders $M$ : Intrauterine transmission and clinical outcome of 248 pregnancies with primary cytomegalovirus infection in relation to gestational age. J Clin Virol 2011;52:244-246.

4 Picone O, Vauloup-Fellous C, Cordier AG, Guitton S, Senat MV, Fuchs F, Ayoubi JM, Grangeot Keros L, Benachi A: A series of 238 cytomegalovirus primary infections during pregnancy: description and outcome. Prenat Diagn 2013;33:751-758.

5 Enders G, Bäder U, Lindemann L, Schalasta G, Daiminger A: Prenatal diagnosis of congenital cytomegalovirus infection in 189 pregnancies with known outcome. Prenat Diagn 2001;21:362-377.

6 Barkovich AJ, Lindan CE: Congenital cytomegalovirus infection of the brain: imaging analysis and embryologic considerations. AJNR Am J Neuroradiol 1994;15:703-715.

7 De Vries LS, Gunardi H, Barth PG, Bok LA, Groenendaal F: The spectrum of cranial ultrasound and magnetic resonance imaging abnormalities in congenital cytomegalovirus infection. Neuropediatrics 2004;35:113-119.
8 Averill LW, Kandula VVR, Akyol Y, Epelman $\mathrm{M}$ : Fetal brain magnetic resonance imaging infection with postnatal imaging correlation. Semin Ultrasound CT MR 2015;36:476-486.

9 Oosterom N, Nijman J, Gunkel J, Wolfs TFW, Groenendaal F, Verboon-Maciolek MA, de Vries LS: Neuro-imaging findings in infants with congenital cytomegalovirus infection: relation to trimester of infection. Neonatology 2015;107:289-296.

10 Revello MG, Gerna G: Diagnosis and management of human cytomegalovirus infection in the mother, fetus, and newborn infant. Society 2002;15:680-715.

11 Prince HE, Lape-Nixon M: Role of cytomegalovirus (CMV) IgG avidity testing in diagnosing primary $\mathrm{CMV}$ infection during pregnancy. Clin Vaccine Immunol 2014;21:13771384.

12 Picone O, Teissier N, Cordier AG, VauloupFellous C, Adle-Biassette H, Martinovic J, Senat MV, Ayoubi JM, Benachi A: Detailed in utero ultrasound description of 30 cases of congenital cytomegalovirus infection. Prenat Diagn 2014;34:518-524.

13 Revello MG, Genini E, Gorini G, Klersy C, Piralla A, Gerna G: Comparative evaluation of eight commercial human cytomegalovirus IgG avidity assays. J Clin Virol 2010;48:255259 .
14 Lazzarotto T, Guerra B, Gabrielli L, Lanari M, Landini MP: Update on the prevention, diagnosis and management of cytomegalovirus infection during pregnancy. Clin Microbiol Infect 2011;17:1285-1293.

15 Korver AMH, de Vries JJC, de Jong JW, Dekker FW, Vossen ACTM, OudesluysMurphy AM: Awareness of congenital cytomegalovirus among doctors in the Netherlands. J Clin Virol 2009;46(suppl 4):S11-S15.

16 Pereboom MTR, Manniën J, van Almkerk KDJ, Spelten ER, Gitsels JT, Martin L, Hutton EK, Schellevis FG: What information do Dutch midwives give clients about toxoplasmosis, listeriosis and cytomegalovirus prevention? An exploratory study of videotaped consultations. Patient Educ Couns 2014;96: 29-35.

17 de Vries JJC, van Zwet EW, Dekker FW, Kroes ACM, Verkerk PH, Vossen ACTM: The apparent paradox of maternal seropositivity as a risk factor for congenital cytomegalovirus infection: a population-based prediction model. Rev Med Virol 2013;23:241-249.

18 Boppana SB, Fowler KB, Britt WJ, Stagno S, Pass RF: Symptomatic congenital cytomegalovirus infection in infants born to mothers with preexisting immunity to cytomegalovirus. Pediatrics 1999;104:55-60.

19 Picone O, Grangeot-Keros L, Senat M, Fuchs F, Bouthry E, Ayoubi J, Benachi A, VauloupFellous C: Cytomegalovirus non-primary infection during pregnancy. Can serology help with diagnosis? J Matern Fetal Neonatal Med 2016;30:224-227.
Antenatal Maternal Serology in

Congenital CMV Infection
Fetal Diagn Ther 2017;42:144-149

DOI: $10.1159 / 000456615$ 